\title{
Effects of the QCD equation of state and lepton asymmetry on primordial gravitational waves
}

\author{
Fazlollah Hajkarim, ${ }^{*}$ Jürgen Schaffner-Bielich, ${ }^{\dagger}$ and Stephan Wystub \\ Institut für Theoretische Physik, Goethe Universität, \\ Max von Laue Strasse 1, D-60438 Frankfurt am Main, Germany \\ Mandy M. Wygas ${ }^{\S}$ \\ Fakultät für Physik, Universität Bielefeld, Postfach 100131, 33501 Bielefeld, Germany
}

(Received 8 April 2019; published 21 May 2019)

\begin{abstract}
Using the quantum chromodynamics (QCD) equation of state from lattice calculations we investigate effects from QCD on primordial gravitational waves produced during the inflationary era. We also consider different cases for vanishing and nonvanishing lepton asymmetry where the latter one is constrained by cosmic microwave background experiments. Our results show that there is up to a few percent deviation in the predicted gravitational wave background in the frequency range around the QCD transition $\left(10^{-10}-10^{-7} \mathrm{~Hz}\right)$ for different lattice QCD equations of state, or at larger frequencies for nonvanishing lepton asymmetry using perturbative QCD. Future gravitational wave experiments with high enough sensitivity in the measurement of the amplitude of primordial gravitational waves like SKA, EPTA, DECIGO, and LISA can probe these differences and can shed light on the real nature of the cosmic QCD transition and the existence of a nonvanishing lepton asymmetry in the early Universe.
\end{abstract}

DOI: 10.1103/PhysRevD.99.103527

\section{INTRODUCTION}

The era of observing the Universe beyond electromagnetic waves began with the first gravitational wave (GW) observation of LIGO produced from two merging black holes [1]. This gives the opportunity to probe phenomena in astrophysics and cosmology which are impossible or difficult to be observed by photons. The inflationary scenario has been proposed in cosmology as a solution to the flatness, the horizon, and magnetic monopole problems [2,3]. It was shown that inflation can also produce primordial gravitational waves (PGWs) as the tensor perturbation of the metric of spacetime [4]. This also opens up a direct way to check the physics of the early Universe before big bang nucleosynthesis $(\mathrm{BBN})$ which until now has been hidden from our sight except for its possible effects on the cosmic microwave background [5-8]. Different phenomena like the electroweak transition, QCD transition, phase transition in the dark sector, early matter domination, etc., can be present before

\footnotetext{
*hajkarim@th.physik.uni-frankfurt.de

sschaffner@astro.uni-frankfurt.de

*wystub@astro.uni-frankfurt.de

${ }^{\S}$ m.wygas@physik.uni-bielefeld.de
}

Published by the American Physical Society under the terms of the Creative Commons Attribution 4.0 International license. Further distribution of this work must maintain attribution to the author(s) and the published article's title, journal citation, and DOI. Funded by SCOAP.
BBN which can produce extra GWs or affect PGWs produced by the inflationary scenario [9-11].

The equation of state (EOS) of the standard model (SM) can have different impacts on PGWs due to possible features coming from the quark-gluon and the electroweak transitions [9-11]. These effects can be measured by future $\mathrm{GW}$ experiments. There are some ongoing and future space and earth based GW detectors like DECIGO [12,13], LISA [14], SKA [15], and EPTA [16], which can measure the possible effects of cosmic (phase) transitions in the visible and dark side of the Universe in the relevant frequency ranges.

The thermal effect of the SM on PGWs appears via the trace anomaly and the energy and entropy density of radiation in the equation of motion for PGWs produced from inflation. The trace anomaly of the energy-momentum tensor of the SM shows deviations of the EOS from pure radiation (with $p=\rho / 3$ ), which are due to quantum effects and the nonrelativistic behavior of SM particles at temperatures below about one third of their masses [9,17-19]. Effects from the trace anomaly in the SM are most pronounced at the QCD transition [20].

The QCD transition can affect the cosmology of the early Universe in different aspects like its effect on the relic density of dark matter and the GW spectrum [9,21-30]. What we know from lattice QCD calculations at vanishing chemical potentials for baryon, electric charge, and strangeness number is that the QCD transition is a smooth 
crossover [20]. This is in contrast to first studies on the QCD phase diagram for the early Universe which adopted a first order or second order phase transition [31,32]. However, the effect of considering nonvanishing chemical potentials can slightly change the strength of the transition and lead to different EOSs compared to the case of vanishing chemical potentials $[25,33]$.

In the present study we focus on the imprints of QCD on PGWs for the cases of vanishing and nonvanishing lepton flavor asymmetry in the early Universe. This paper is organized as follows. In Sec. II we outline our formalism to compute the relic PGW spectrum and the relevant thermodynamic relations. Then we discuss the impact of different QCD EOSs, based on different lattice QCD results, on the PGW spectrum in Sec. III, paying special attention to effects from the charm quark contribution. Effects on PGWs from nonvanishing chemical potentials, in particular, from a nonvanishing lepton asymmetry, are discussed in Sec. IV. Finally, we conclude in Sec. V.

\section{PRIMORDIAL GRAVITATIONAL WAVES FROM INFLATION}

The production of GWs by the inflationary scenario in the early Universe can be considered by doing a perturbative analysis of the Friedmann equations. In standard cosmology the following metric describes the evolution of the cosmos assuming vanishing curvature which is a reasonable assumption for an isotropic and homogeneous universe [34] and matches with observations [8]:

$$
d s^{2}=-d t^{2}+a(t)^{2} d \mathbf{x}^{2},
$$

where the relation between the cosmic time $t$ and the conformal time $\eta$ can be defined by $d t=a d \eta$. The tensor perturbation equation in the Fourier space which shows the evolution of PGWs is given by [34]

$$
h^{\prime \prime}(k, \eta)+2 \mathcal{H}(\eta) h^{\prime}(k, \eta)+k^{2} h(k, \eta)=0,
$$

where ${ }^{\prime}:=d / d \eta$. The conformal Hubble rate is denoted by $\mathcal{H}=a^{\prime} / a=a H$. By using $v(k, \eta)=a(\eta) h(k, \eta)$ one has

$$
v^{\prime \prime}(k, \eta)+\left(k^{2}-\frac{a^{\prime \prime}}{a}\right) v(k, \eta)=0,
$$

with

$$
\frac{a^{\prime \prime}}{a}=\frac{4 \pi G}{3}\left(\rho_{\mathrm{tot}}-3 p_{\mathrm{tot}}\right),
$$

where $8 \pi G=1 / M_{\mathrm{Pl}}^{2}$ and $M_{\mathrm{Pl}}=1.22 \times 10^{19} \mathrm{GeV}$. The quantity in the parentheses at the right-hand side of Eq. (4) is called the trace anomaly (or interaction measure) and can be written as follows [35-37]:

$$
\frac{I(T)}{T^{4}}=\frac{\rho_{\mathrm{tot}}-3 p_{\mathrm{tot}}}{T^{4}}=T \frac{\partial}{\partial T}\left(\frac{p_{\mathrm{tot}}}{T^{4}}\right)_{\mu / T} .
$$

In Eq. (4) one should consider the total energy and pressure density with respect to the scale factor taking into account entropy conservation in the early Universe. The entropy density $s_{\text {tot }}$ can be derived by using thermodynamic relations which we show below. For this, one needs also the Friedmann equation which reads

$$
H^{2}=\frac{8 \pi G}{3} \rho_{\mathrm{tot}} .
$$

At any specific time, $t(\eta)$, during the cosmic evolution superhorizon modes can be defined for $k \eta \ll 1$. When the Universe expands and modes enter the horizon, they are identified as subhorizon modes by $k \eta \gg 1$. The frequency of each mode $k$ can be written as $f=k / 2 \pi$. The initial condition for modes outside the horizon that we used to solve Eq. (3) to compute the GW spectrum are $[18,34]$

$$
\begin{aligned}
v\left(k, \eta_{\text {ini }}\right) & =\frac{1}{k^{3 / 2}}, \\
v^{\prime}\left(k, \eta_{\text {ini }}\right) & =\frac{v\left(k, \eta_{\text {ini }}\right)}{r_{\text {ini }}}, \quad r_{\text {ini }}=\frac{1}{a\left(\eta_{\text {ini }}\right) H\left(\eta_{\text {ini }}\right)},
\end{aligned}
$$

where the oscillatory factor of the wave function, $\exp (i k \eta)$, is neglected, as it only affects the phase of the GW, not the amplitude we are interested in. In choosing these initial conditions, only the $k$ dependence is important for our purpose.

By assuming entropy conservation during the QCD transition and until today, one can compute the evolution of $\rho_{\text {tot }}(a)$ by solving Eq. (6) backward in time, i.e., from today $\left(a_{0}=1\right)$ to a chosen scale factor $a$ in the early Universe [38]. Then the solution can be used to solve Eq. (3).

Because of the large range of the scale factor from today $\left(a_{0}=1\right)$ back to the early Universe when we consider modes well before horizon crossing (temperatures well above the electroweak transition) numerical problems for solving the differential equation given by Eq. (3) might arise. Therefore, one solves the differential equation either until horizon crossing for each mode or until a temperature after neutrino decoupling includes all the evolution of the EOS in the calculation. In practice both of these ways of calculating the GW spectrum will give approximately the same final result. Since the slight change of the EOS due to the change of trace anomaly happens in a short interval of the scale factor with a tiny deviation from the radiation-like EOS, this will cause a tiny change in the amplitude of the GW when the mode enters the horizon until the end of neutrino decoupling. This procedure is sufficient for our goal to show the effects from QCD and from a lepton asymmetry on PGWs. We also check the difference between two procedures in a specific frequency $\left(3 \times 10^{-11}\right.$ and 
$5 \times 10^{-8} \mathrm{~Hz}$ ) range such that we can find the numerical solution in a precise way. Definitely, if one finds any evidence of PGWs in experiments, a more detailed calculation can be done by fixing the scale of inflation from the data to have a more precise handle on the aforementioned effects to match theory with experiment.

For each polarization mode $(\lambda)$ of the GW, Eq. (2) is valid and the amplitude of perturbations can be written as [19]

$$
h_{\lambda}(k, \eta)=h_{\lambda}^{\text {prim }}(k) Y(\eta, k)=\frac{v(k, \eta)}{a(\eta)}
$$

then the energy density of GWs is given by $[17,19]$

$$
\begin{gathered}
\rho_{\mathrm{GW}}(\eta)=\frac{M_{\mathrm{Pl}}^{2}}{32 \pi a(\eta)^{2}}\left\langle h_{i j}^{\prime}(k, \mathbf{x}) h^{i j^{\prime}}(k, \mathbf{x})\right\rangle, \\
\left\langle h_{i j}^{\prime}(k, \mathbf{x}) h^{i j \prime}(k, \mathbf{x})\right\rangle=\int \frac{d k}{k} \mathcal{P}_{T}(k, \eta) .
\end{gathered}
$$

The tensor power spectrum is defined by

$$
\begin{aligned}
\mathcal{P}_{T}(k, \eta) & =\frac{k^{3}}{\pi^{2}} \sum_{\lambda}\left\langle\left|h_{\lambda}(k, \eta)\right|^{2}\right\rangle \\
& =\mathcal{P}_{T}^{\text {prim }}(k)[Y(k, \eta)]^{2},
\end{aligned}
$$

where its time independent part reads

$$
\mathcal{P}_{T}^{\text {prim }}(k)=\frac{k^{3}}{\pi^{2}} \sum_{\lambda}\left\langle\left|h_{\lambda}^{\text {prim }}(k)\right|^{2}\right\rangle=\frac{1}{\pi}\left(\frac{4 H_{\text {inf }}}{M_{\mathrm{Pl}}}\right)^{2} .
$$

The Hubble parameter at the inflation scale is fixed by $H_{\text {inf }}^{2} \approx\left(8 \pi / 3 M_{\mathrm{Pl}}^{2}\right) V_{\text {inf }}$. The relic density of GWs can be obtained by

$$
\Omega_{\mathrm{GW}}(k, \eta)=\frac{\mathcal{P}_{T}^{\text {prim }}(k)}{12 a(\eta)^{2} H(\eta)^{2}}\left[Y^{\prime}(k, \eta)\right]^{2} .
$$

Equations (12) and (13) show that the absolute value of the relic density of PGWs depends on the inflationary scale. Assuming an inflationary scale of $V_{\text {inf }}^{1 / 4}=1.5 \times 10^{16} \mathrm{GeV}$ the relic density of GWs for the frequency range between $10^{-9}-10^{-10} \mathrm{~Hz}$ will be $\Omega_{\mathrm{GW}} h^{2} \sim 2.4 \times 10^{-16}$ [26].

At the horizon it can be found that $\left[Y^{\prime}(k, \eta)\right]^{2}=$ $k^{2}[Y(k, \eta)]^{2}$. This can change when modes come well inside the horizon [17,26]. Since our goal is to evaluate the effect of the EOS on the PGWs and to compare their relic amplitude at high frequency to their relic amplitude at low frequency, using Eqs. (8), (12), and (13), we can write

$$
\Omega_{\mathrm{GW}}\left(k, \eta_{0}\right) \propto \Omega_{\mathrm{GW}}\left(k, \eta_{\mathrm{hc}}\right) \propto k^{5}\left|v\left(k, \eta_{\mathrm{hc}}\right)\right|^{2},
$$

where the horizon crossing mode can be identified by

$$
k=a\left(\eta_{\mathrm{hc}}\right) H\left(\eta_{\mathrm{hc}}\right) .
$$

The temperature at horizon crossing can also be determined by using Eqs. (6) and (15). One can also find the following approximate relation between $\mathrm{PGW}$ relic, energy, and entropy density at horizon crossing $[9,17,26]$

$$
\Omega_{\mathrm{GW}}\left(k, \eta_{0}\right) \propto \rho_{\mathrm{tot}}\left(T_{h c}\right) s_{\mathrm{tot}}\left(T_{\mathrm{hc}}\right)^{-4 / 3} .
$$

We can solve Eqs. (3), (4), and (6) with the initial conditions given by Eq. (7) until the scale factor at horizon reaches a value where each mode $k$ crosses the horizon or until a scale factor crosses at lower temperatures, e.g., after neutrino decoupling. After neutrino decoupling, since the GWs evolve like radiation $\left(\rho_{\mathrm{GW}} \propto a^{-4}\right)$ in case of the absence of any phase transition afterwards, the spectrum will be unchanged until today except for the damping in the amplitude due to the expansion. This helps us to pin down the relative difference of PGWs for different modes due to the evolution of the EOS with temperature in the early Universe. We do not consider the effect of an anisotropic stress due to the free streaming of photons and decoupled neutrinos, which appears as a source on the right-hand side of Eq. (2), because it is effective only for frequencies smaller than $\sim 5 \times 10^{-11} \mathrm{~Hz}[17,26,39]$.

\section{THE ROLE OF THE EQUATION OF STATE OF THE SM ON PGWS}

In order to solve Eq. (3) for different GW wave numbers $k$ it is required to first solve Eqs. (4) and (6) to find the temperature as a function of $a$ or $\eta$. For this purpose, we should know the quantities $\rho_{\text {tot }}$ and $p_{\text {tot }}$ at each temperature $T$. The total energy and pressure density can be computed from the following equations [40]:

$\rho_{\mathrm{tot}}(T, \mu)=\sum_{i} \frac{g_{i}}{2 \pi^{2}} \int_{m_{i}}^{\infty} d E \times E^{2} \sqrt{E^{2}-m_{i}^{2}}\left(\frac{1}{e^{\frac{E-\mu_{i}}{T}} \pm 1}\right)$,

$p_{\text {tot }}(T, \mu)=\sum_{i} \frac{g_{i}}{6 \pi^{2}} \int_{m_{i}}^{\infty} d E\left(E^{2}-m_{i}^{2}\right)^{3 / 2}\left(\frac{1}{e^{\frac{E-\mu_{i}}{T}} \pm 1}\right)$,

with the sum over all particle species $i$ with degrees of freedom $g_{i}$ and chemical potential $\mu_{i}$. The total entropy density is given by

$T s_{\text {tot }}(T, \mu)=\rho_{\text {tot }}(T, \mu)+p_{\text {tot }}(T, \mu)-\sum_{i} \mu_{i} n_{i}\left(T, \mu_{i}\right)$.

For each particle species the net number density of particles minus antiparticles can be defined as 


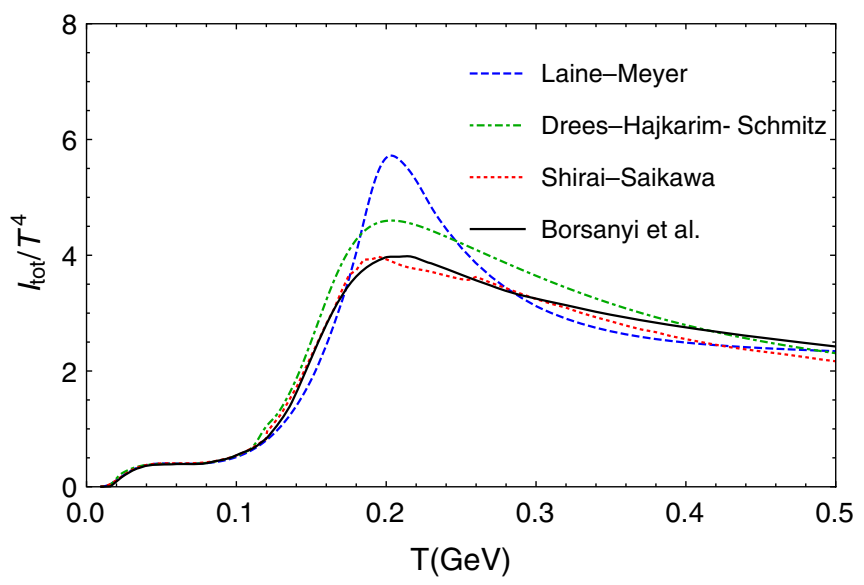

FIG. 1. The trace anomaly including lattice QCD results for temperatures up to $500 \mathrm{MeV}$ taken from different approaches $[21,26,28,42]$. See text for details.

$$
\begin{aligned}
n_{i}\left(T, \mu_{i}\right)= & \frac{g_{i}}{2 \pi^{2}} \int_{m_{i}}^{\infty} d E E \sqrt{E^{2}-m_{i}^{2}} \\
& \times\left(\frac{1}{e^{\frac{E-\mu_{i}}{T}} \pm 1}-\frac{1}{e^{\frac{E+\mu_{i}}{T}} \pm 1}\right) .
\end{aligned}
$$

The above equations can be used to determine the energy and entropy density of the SM which can be implemented in Eqs. (3), (4), and (6) to compute the relic density of PGW in the early Universe according to Eq. (13).

In this section we only consider the case of vanishing chemical potentials. Several studies have been performed before for the case of vanishing chemical potentials using different lattice QCD results around the QCD transition available at that time [21,26-28,41,42] and considering different assumptions for the EOS in the perturbative regime of QCD, at the electroweak transition, and for neutrino decoupling.

As shown in Refs. [18,26] the characteristic frequency of PGWs related to the QCD transition temperature, $T_{\mathrm{QCD}} \sim 150 \mathrm{MeV}$, is $f_{\mathrm{QCD}} \approx 3 \times 10^{-9} \mathrm{~Hz}$. The effect of neutrino decoupling at low temperature $(T \sim 1 \mathrm{MeV}$ and $f \sim 10^{-11} \mathrm{~Hz}$ ) is important to compute the precise value of temperature with respect to the scale factor [43]. This effect appears due to the varying temperatures of different neutrino flavors during and after neutrino decoupling [43].

The main QCD EOS we use is the one by Ref. [28] (labeled as "Borsanyi et al." in the figures). In Ref. [28] the EOS from $10 \mathrm{MeV}$ to $(500 \mathrm{MeV}) 1 \mathrm{GeV}$ for $(2+1) 2+$ $1+1$ flavors by using lattice methods is computed. Their computed trace anomaly for all SM particles and the predicted PGW spectrum are shown in Figs. 1 and 2, respectively. By using hard thermal loop corrections and the perturbative QCD approach up to order $\mathcal{O}\left(g_{s}^{6}\right)$ including effects from charm and bottom quarks, they derive the EOS for temperatures above $1 \mathrm{GeV}[44,45]$. They use the results of Ref. [42] for very high temperatures around the

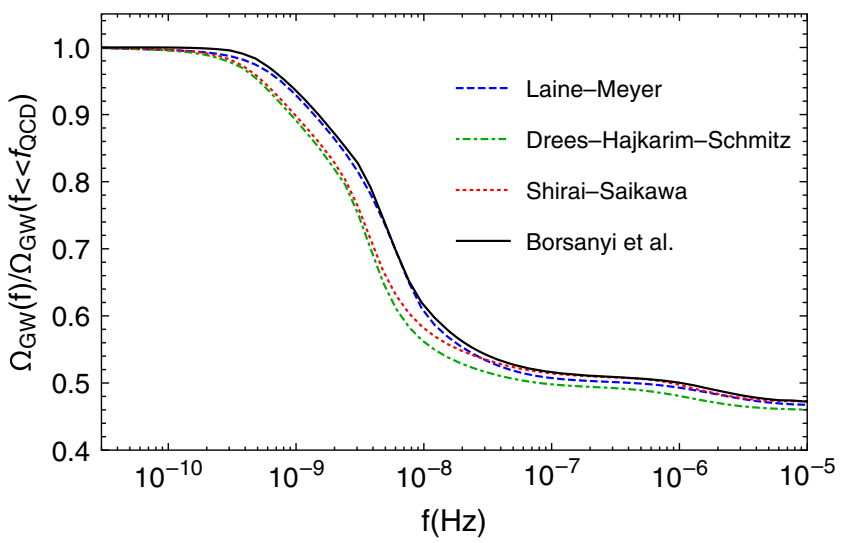

FIG. 2. The PGW spectrum with respect to frequency using different EOSs for the early Universe shown in the previous figure.

electroweak transition. For smaller temperatures they adopt a hadron resonance gas (HRG) model approach [46]. Thereby they provide the EOS for temperatures between $1 \mathrm{MeV}$ and $100 \mathrm{GeV}$ including all SM particles. The dataset of Ref. [28] is the one we use to extract the impact of charm quarks from lattice QCD calculations on PGWs.

In Figs. 1 and 2 the corresponding result of [21] for the QCD EOS of the standard model is shown using the data of the HotQCD Collaboration [36] for $2+1$ flavors and the EOS including also the charm quark by Ref. [47]. Also, the HRG model data is used for temperatures between 70 and $100 \mathrm{MeV}$ [46]. The remaining SM particles are assumed to be free particles. Moreover, the effect of neutrino decoupling has been considered using Ref. [43]. The interpolated result for the EOS of Ref. [37], using another lattice calculation for vanishing chemical potentials, highly matches the result of [36] in the temperature range of 130-230 MeV, so that we do not include their results in this paper.

Additionally, we compare the thermal evolution of the EOS of the SM with a calculation by Laine and Meyer [42] who used the older treatment of Laine and Schroeder [41] for temperatures below $110 \mathrm{GeV}$. In Ref. [41] the radiative corrections up to order $\mathcal{O}\left(g_{s}^{2}\right)$ for a running quark mass are considered. For temperatures below $350 \mathrm{MeV}$ old lattice data for pure glue theory is used. The EOS of Ref. [41] includes temperatures between $10 \mathrm{MeV}$ and $1 \mathrm{TeV}$ including all SM particles. However, since the mass of the Higgs boson was unknown at that time, they have considered a different value from the nowadays accepted one. This issue is fixed in the work of Laine and Mayer [42] by considering $125 \mathrm{GeV}$ for the Higgs mass and including corrections from lattice field theory calculations around the electroweak transition $(110 \mathrm{GeV} \lesssim T \lesssim 250 \mathrm{GeV})$. For temperatures above $250 \mathrm{GeV}$ they have considered the perturbative result of [48]. The result for their treatment for the trace anomaly up to a temperature of $500 \mathrm{MeV}$ is shown in Fig. 1. The comparison of the influence of the EOS of Laine 
and Meyer on the PGW spectrum with the other EOSs for the early Universe is shown in Fig. 2.

Another treatment of the SM EOS that we investigate is from the work of Ref. [26] which used lattice QCD results for $2+1+1$ flavors [28] matched to a HRG model [46] at temperatures below the QCD transition and perturbative QCD up to $\mathcal{O}\left(g_{s}^{6} \log g_{s}\right)$ at higher temperatures [45]. For temperatures around the electroweak transition the results of Refs. $[42,49,50]$ considering lattice calculations and perturbative calculations are used. At very low temperatures around electron and neutrino decoupling, the result of [51] is adopted to consider the effect of neutrino oscillations. The effect of this EOS on the trace anomaly, PGW, and the differences compared to result obtained with the EOS of Ref. [28] are shown in Figs. 1 and 2, respectively.

In Fig. 1 results for the trace anomaly of QCD based on different lattice calculations reported in the literature are shown. It can be seen that the location of the peak in the trace anomaly is similar but the height differs for different approaches as different input from lattice calculations have been used. Figure 2 shows the effect of using the different results for the trace anomaly on the relic density of PGWs as a function of the frequency. For frequencies in the range $10^{-10}-10^{-9} \mathrm{~Hz}(T \sim 10-100 \mathrm{MeV}$ for horizon crossing) the HRG model and muons play the major role for the PGW relic density. For frequencies between $10^{-9}$ and $10^{-7} \mathrm{~Hz}$ the EOS including lattice QCD results, tau leptons, and bottom quarks are important for determining the GW relic density for horizon crossing temperatures between $100 \mathrm{MeV}$ and $10 \mathrm{GeV}$. For temperatures around $100 \mathrm{GeV}$ and frequencies around $10^{-6} \mathrm{~Hz}$ the appearance of top quarks and the electroweak sector including $\mathrm{W}^{ \pm}, \mathrm{Z}$, and Higgs bosons have the dominant impact on the SM EOS and the prediction of the stochastic GW background.

Two of the EOSs [21,26] shown in Fig. 2 consider effects from neutrino decoupling but this effect is not taken into account for the other EOSs [28,42]. Neutrino decoupling leads to a shift of the PGW spectrum to higher frequencies compared to the case without considering neutrino decoupling taken into account. Moreover, it causes a relative error of up to $\sim 10 \%$ between two cases as shown in Fig. 3. The discrepancy originates mainly from the change in determining the precise relation between the scale factor and the temperature using Eqs. (4) and (6) which differs if one takes into account neutrino decoupling or not. The differences in the trace anomaly (shown in Fig. 1), energy, and entropy density are mostly due to the various treatments of the QCD EOS. As Fig. 3 shows, using different EOSs results in a deviation of up to $3 \%-4 \%$ at frequencies around $3 \times 10^{-9} \mathrm{~Hz}$ and up to $3 \%$ for higher frequencies if one neglects the effect from neutrino decoupling. The deviations in the predicted PGW relic shown in Fig. 2 are computed at the scale factor of horizon crossing for each mode which is numerically doable. We also studied the

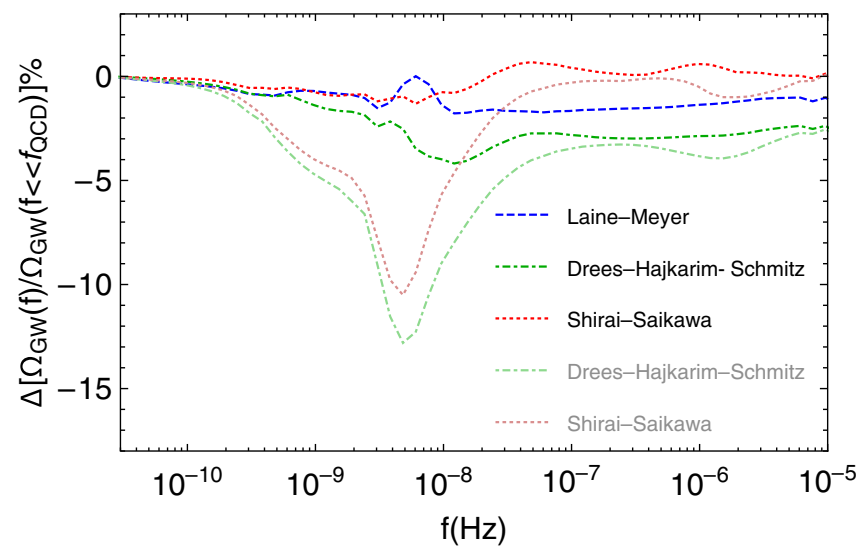

FIG. 3. The relative differences of the predicted relic density of PGWs using the EOSs from Refs. [21,26,42] compared to the one of Ref. [28]. The opaque curves do not include the effect of electron and neutrino decoupling to better compare the role of QCD on the relic density of PGWs with the main data set [28]. The transparent curves include the contribution due to electron and neutrino decoupling.

deviations for a limited frequency range $\left(3 \times 10^{-11}\right.$ and $\left.5 \times 10^{-8} \mathrm{~Hz}\right)$ at a fixed scale factor after neutrino decoupling when the evolution of the EOS will not be affected by SM particles any more. Our results show that highly evolving the EOS especially around the QCD transition improve the predicted PGW relic around 1\%, since the deviation of the EOS from radiation causes a small damping of PGWs after horizon crossing. We do not show the plot for this calculation in this paper, since our frequency range is limited and doing it for a larger frequency range is numerically expensive. These discrepancies between different treatments of the EOS can be distinguished by SKA, EPTA, LISA, and DECIGO at different frequencies by the observation of PGWs.

In Fig. 4 the effect of considering charm quarks in lattice calculations on the predicted relic density of PGWs is shown using lattice data from Ref. [28] for $2+1$ and $2+$ $1+1$ flavors. The difference is small but not negligible. The relative difference of the predicted pattern of PGWs between $2+1$ and $2+1+1$ flavors in lattice QCD for different frequencies is shown in Fig. 5 and amounts up to $2.6 \%$. For frequencies higher than $2 \times 10^{-8} \mathrm{~Hz}$ the difference is due to the change in the relation between the energy density and scale factor computed from Eq. (6), since a lower temperature as an initial condition affects this relation for the higher temperature.

We also consider the uncertainties of the lattice data and the perturbative QCD calculations up to $\mathcal{O}\left(g_{s}^{6}\right)$ for the temperature range of $100 \mathrm{MeV}$ to $10 \mathrm{GeV}$ considering $2+$ $1+1+1$ flavors, i.e., including effects from bottom quarks, which is discussed in Ref. [28]. The resulting error band in the relic density of PGWs is depicted in Fig. 4. One sees that the changes are small. In fact, the relative error in the relic density of PGWs amounts to a discrepancy 


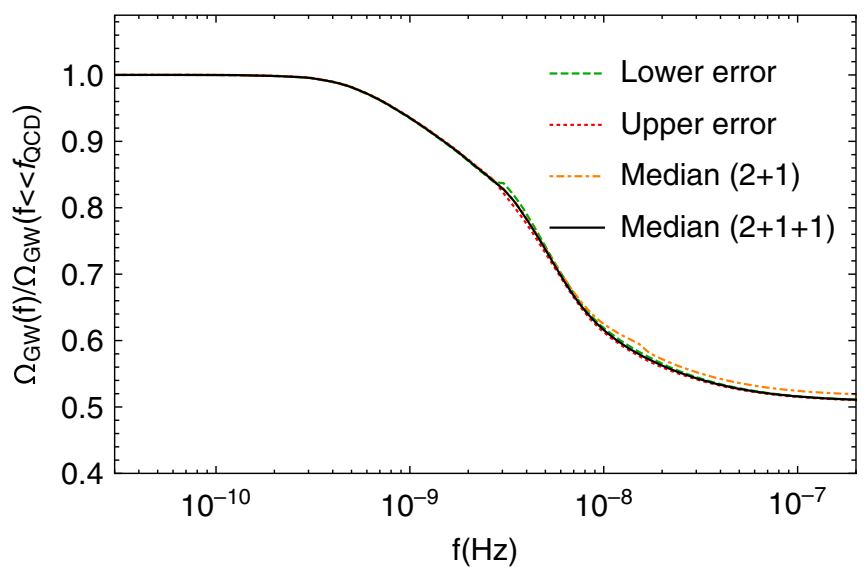

FIG. 4. The error band of the relic density of PGWs from the median value $(2+1+1)$ by taking the lower and upper bounds from the lattice data of Ref. [28]. Also, the effect of including charm quarks in lattice simulations on the relic density is presented using the data of Ref. [28] for $2+1$ and $2+1+1$ flavors for the median values. As it can be seen, there is a deviation between the computed relic density of PGWs due to charm quarks mostly for frequencies around $10^{-8} \mathrm{~Hz}$ and higher.

of at most $1.1 \%$ around the QCD transition as shown in Fig. 6.

\section{SM WITH NONVANISHING CHEMICAL POTENTIALS AND PGWs}

The value of the lepton asymmetry in the Universe is constrained by analyses of BBN and the cosmic microwave background to be [52]

$$
l=\frac{n_{L}}{s} \lesssim 0.012 .
$$

Also from the Planck data [53] one knows the amount of baryon asymmetry of the Universe

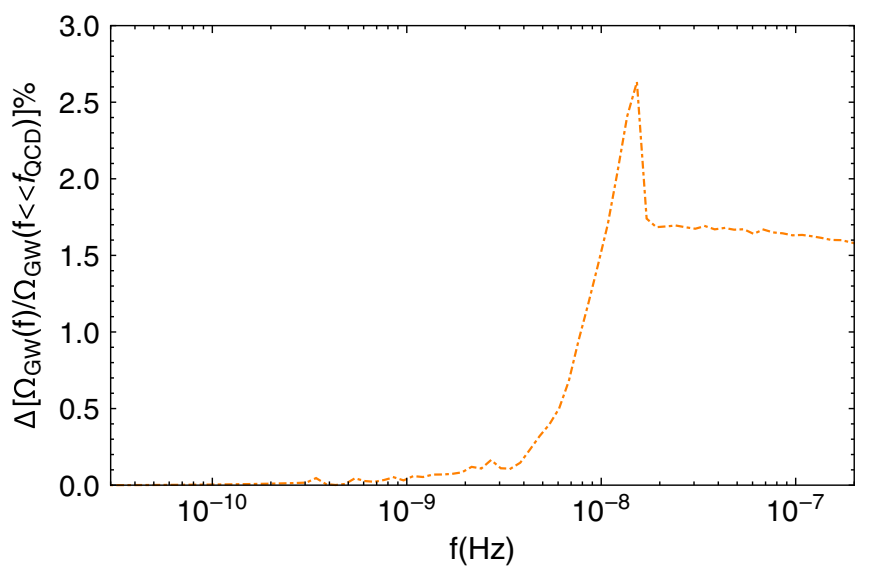

FIG. 5. The error due to the effect of charm quarks at low temperatures in lattice simulations on PGWs is presented using the data of Ref. [28] for $2+1$ and $2+1+1$ flavors.

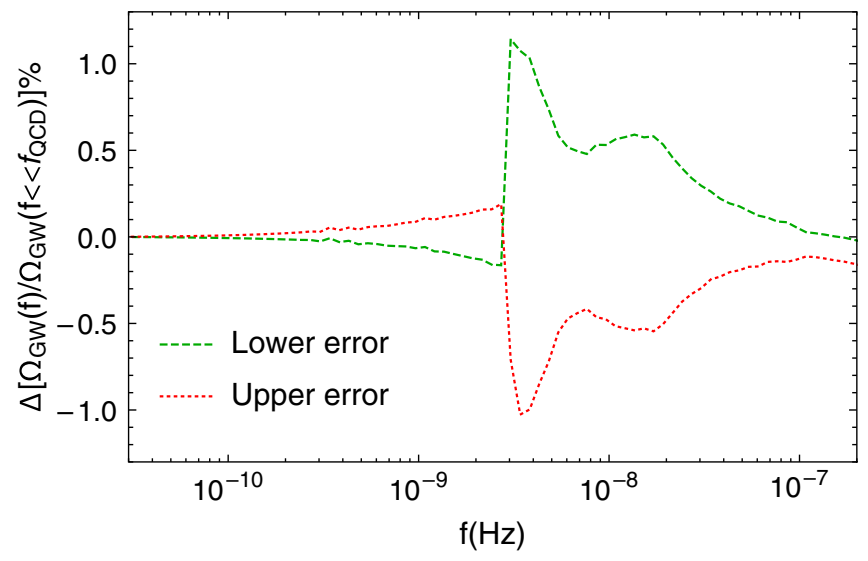

FIG. 6. The relative error on the relic density of PGWs due to the uncertainties in the EOS from lattice QCD and perturbative calculations from Ref. [28].

$$
b=\frac{n_{B}}{s} \approx 8 \times 10^{-11} .
$$

Considering SM particles in a thermal bath and assuming that sphaleron processes occur efficiently, then the lepton asymmetry is related to the baryon asymmetry by $l=-\frac{51}{28} b$ [54]. Such tiny values for the lepton asymmetry and baryon asymmetry do not lead to a first order phase transition of QCD $[55,56]$. However, such a small value of $l$ has not been confirmed experimentally. The effect of a sizable lepton asymmetry on the evolution of the chemical potentials of SM particles with respect to temperature and its effect on the cosmic trajectory has been investigated in Refs. [33,55].

In the early Universe, between neutrino oscillations $\left(T_{\mathrm{osc}} \sim 10 \mathrm{MeV}\right)$ and the electroweak transition $\left(T_{\mathrm{ew}} \sim\right.$ $100 \mathrm{GeV}$ ), conservation of nonvanishing lepton flavor asymmetries, baryon asymmetry, and electric charge leads to the following set of equations $[33,55]$ :

$$
\begin{aligned}
l_{f} s(T, \mu) & =n_{f}\left(T, \mu_{f}\right)+n_{\nu_{f}}\left(T, \mu_{\nu_{f}}\right), \quad f=e, \mu, \tau, \\
b s(T, \mu) & =\sum_{i} b_{i} n_{i}\left(T, \mu_{i}\right), \\
0 & =\sum_{i} q_{i} n_{i}\left(T, \mu_{i}\right),
\end{aligned}
$$

where $n_{i}$ is the net number density of particles minus antiparticles given by Eq. (20) and we presumed electric charge neutrality of the Universe in the last equation.

The total entropy density can be determined according to Eq. (19) using Eqs. (17) and (18) considering all relevant SM particles and their chemical potentials. Solving this system of coupled equations at a given temperature, we get the temperature evolution of the SM chemical potentials [55] and thus we can compute the total pressure and energy density for nonvanishing lepton asymmetries (cf. [57]). For the numerical evaluation we assumed equally distributed lepton flavor asymmetries, $l_{f}=l / 3$. 
For different temperature ranges one can find approximate relations between the lepton flavor chemical potentials and the electric charge chemical potential. For example, in the temperature range where the lattice QCD plays a major role, i.e., $150 \mathrm{MeV}<T<350 \mathrm{MeV}$ (the upper bound is defined due to the presence of charm quarks at higher temperatures) we have $\mu_{Q} \approx \mu_{l_{f}} / 2$. For temperatures between the QCD transition and the temperature where pions are not relativistic anymore $\left(m_{\pi^{ \pm}} / 3<T<150 \mathrm{MeV}\right)$ one finds $\mu_{Q} \approx 2 \mu_{l_{f}} / 3$. For even lower temperatures but above neutrino decoupling one can find $\mu_{Q} \approx \mu_{l_{f}}$ due to charge conservation $[33,55,58]$.

To calculate the influence of a nonvanishing lepton asymmetry on PGWs one needs the temperature evolution of the energy and entropy density at nonvanishing lepton asymmetry for the early Universe. We calculate the EOS between 150 and $350 \mathrm{MeV}$ according to Refs. [55,57] using lattice QCD susceptibilities $\left[\chi_{a b}=\left.\left(\partial^{2} p / \partial \mu_{a} \partial \mu_{b}\right)\right|_{\mu=0}\right]$ $[59,60]$ to determine the evolution of the chemical potentials at nonvanishing lepton asymmetry. The HRG model is computed by using a similar approach as in [46] for temperatures below $100 \mathrm{MeV}$ by considering hadrons up to a mass of $2.5 \mathrm{GeV}$ as an ideal gas. The energy and entropy density at nonvanishing chemical potentials for temperatures above $350 \mathrm{MeV}$ are calculated as described before according to [55], using the results of [41] for considering perturbative QCD effects up to order $\mathcal{O}\left(g_{s}^{2}\right)$ in the case of vanishing chemical potentials.

Figures 7 and 8 show the trace anomaly and the relic density of PGWs for different values of the lepton asymmetry in the early Universe, respectively. There is up to a $10 \%$ difference between considering nearly vanishing $\left(l=-\frac{51}{28} b\right)$ and nonvanishing lepton asymmetry $(l=$ $\left.0.012, l_{e}=l_{\mu}=l_{\tau}=0.004\right)$. This result is based on the computation at the horizon crossing. We also checked it with the calculation at a specific scale factor after neutrino

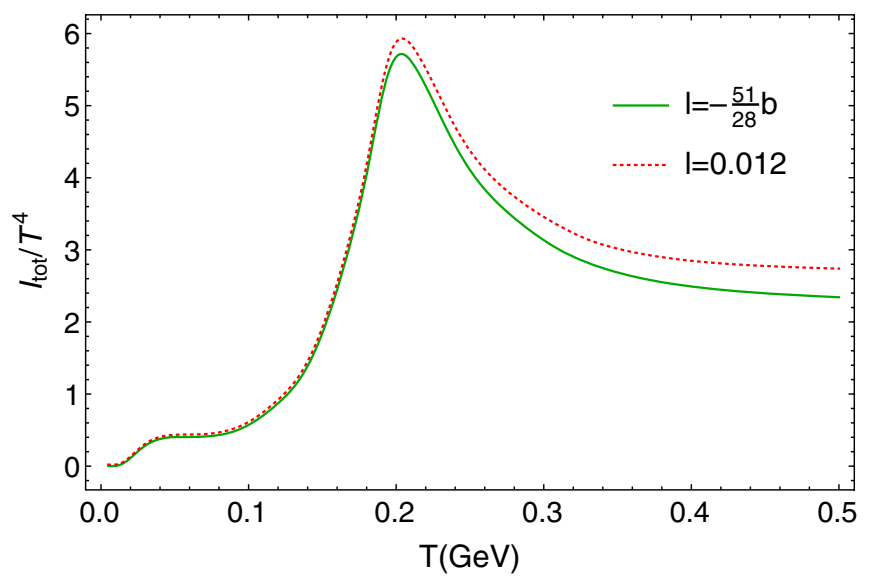

FIG. 7. The trace anomaly for vanishing and nonvanishing lepton asymmetry.

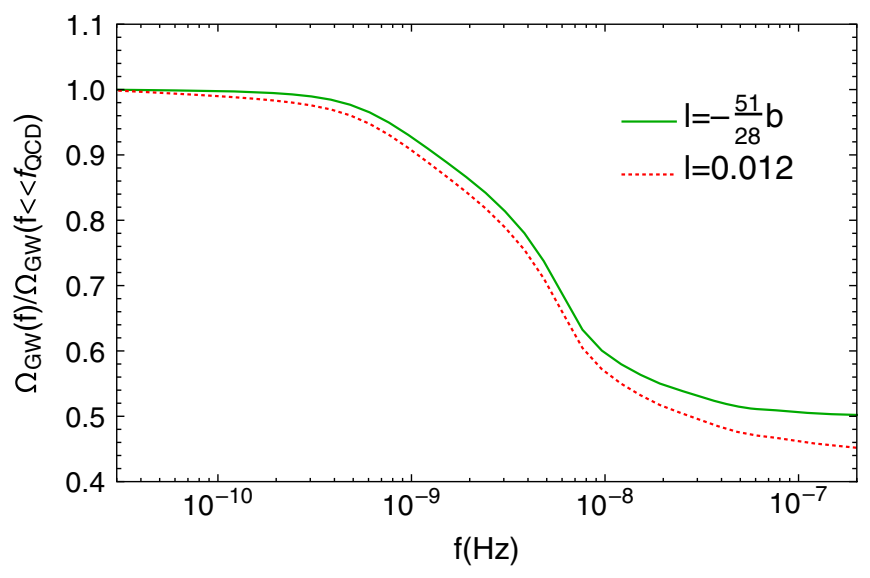

FIG. 8. The scaled relic density of PGWs versus frequency assuming different values for the lepton asymmetry.

decoupling and found the difference between these two methods to be less than $1 \%$. Here we used the EOS calculated according to [57] in the PGWs relic density for frequencies above $\sim 10^{-11} \mathrm{~Hz}$. A deviation of the EOS from the predicted value for vanishing lepton asymmetry can be measured in the spectrum of PGWs for frequencies around $10^{-9}-10^{-6} \mathrm{~Hz}$ by SKA or EPTA, at higher frequencies $10^{-5}-10^{-2} \mathrm{~Hz}$ by LISA (this frequency range is outside the range plotted in Fig. 9), or at $10^{-3}-1 \mathrm{~Hz}$ by DECIGO. The detection of a sizeable lepton asymmetry in the early Universe can give impetus for possible scenarios for the explanation of the matter-antimatter asymmetry in the early Universe and today. We would like to emphasize that such a small deviation in the EOS of the SM from a vanishing lepton asymmetry cannot be observed by cosmic microwave background measurements, since its presence mostly shows up before $\mathrm{BBN}$ when more SM particles are present in the thermal bath.

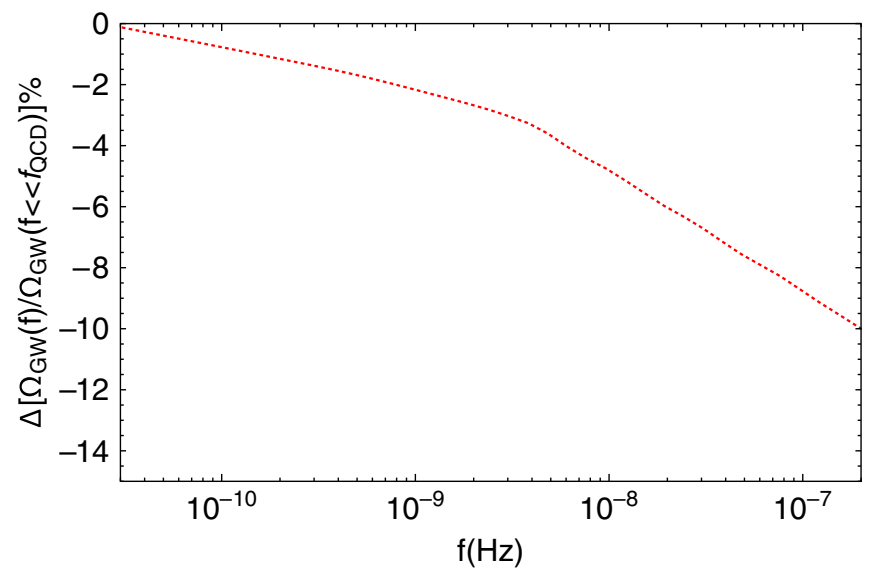

FIG. 9. The relative change of the relic density of PGWs due to the presence of nonvanishing lepton chemical potentials for the SM in the thermal bath of the early Universe using the data of Fig. 8. 


\section{SUMMARY AND CONCLUSIONS}

In this paper we studied the effect of the QCD EOS using different lattice QCD simulations including vanishing and nonvanishing chemical potentials $[28,36,42,47,55]$ on the relic density of PGWs produced by the inflationary scenario. These kind of GWs can be observed by different experiments at a level of less than $1 \%$ in the relic density per frequency depending on the length of observation and the sensitivity [12-16]. The SKA and EPTA experiments are designed for frequencies $10^{-9}-10^{-6} \mathrm{~Hz}$ by measuring the variation in the distance of pulsars. SKA can observe the PGW background relic density for values as small as $\Omega_{\mathrm{GW}} h^{2} \approx 10^{-16}$ depending on the time of exposure which is at the order of few decades. Other experiments like LISA and DECIGO which are proposed for larger frequencies $10^{-5}-1 \mathrm{~Hz}$ can also probe the QCD effects mostly due to perturbative effects on the EOS of the SM at higher temperatures.

Different sets for the EOS from Refs. [21,26,28,42] have been used to calculate the PGW spectrum. The difference between the various EOSs of the SM, using different lattice QCD data as input, results in a relative difference of up to $4 \%$ (Fig. 3) in the relic density of PGWs, mostly between frequencies of $10^{-9}-10^{-8} \mathrm{~Hz}$ and somewhat smaller differences at higher frequencies. We also considered the uncertainties of the EOS given in [28] by taking lower and upper error bounds of lattice QCD calculations into account which leads to a deviation of up to $1.1 \%$ (Fig. 6) in the relic density of PGWs in the frequency range of $10^{-9}-10^{-7} \mathrm{~Hz}$. Additionally, we investigated the effect of considering charm quarks using lattice QCD data at temperatures lower than $1 \mathrm{GeV}$ which causes up to $2.6 \%$ deviation in the predicted PGW density around a frequency of $10^{-8} \mathrm{~Hz}$ (Fig. 5).

We also discussed the effect of a nonvanishing lepton asymmetry on the EOS of matter in the early Universe.
Our calculation shows that this can lead to a difference of up to $2 \%-10 \%$ in the relic density of PGWs for frequencies around and larger than $f_{\mathrm{QCD}} \sim 3 \times 10^{-9} \mathrm{~Hz}$ (Fig. 8). Observing such a deviation from the standard PGW relic density at vanishing lepton asymmetry will elucidate and shed new light on possible solutions for the existence of the baryon asymmetry of the Universe.

Finally, based on our current knowledge of the QCD phase diagram, the presence of uncertainties in lattice simulations, and our ignorance about the properties of the quark-gluon plasma in the early Universe we do not have a unique and confirmed picture about the real nature of QCD at early eras. The observation of stochastic GWs produced by inflation may illuminate these issues and deepen our understanding about the EOS of matter before $\mathrm{BBN}$. The structure of the QCD phase can also be changed due to nonvanishing isospin chemical potential of charged pions or other SM particles and may lead to pion condensation $[61,62]$ which might affect the thermal history of the Universe and also the PGWs. We leave this investigation for future work.

\section{ACKNOWLEDGMENTS}

We thank Dominik Schwarz and Dietrich Bödeker for useful discussions and also Pasi Huovinen and Zoltan Fodor for providing the data of their equations of state. F. H. is grateful to Ken'ichi Saikawa for clarifications related to his work, Ref. [26], and Nicolas Bernal for useful discussions. M. M.W. acknowledges the support by Studienstiftung des Deutschen Volkes. All authors acknowledge support by the Deutsche Forschungsgemeinschaft (DFG, German Research Foundation) through the CRCTR 211 "Strong-interaction matter under extreme conditions" Project No. B315477589-TRR 211.
[1] B. P. Abbott et al. (LIGO Scientific and Virgo Collaborations), Phys. Rev. Lett. 116, 061102 (2016).

[2] A. D. Linde, Phys. Lett. 108B, 389 (1982); Adv. Ser. Astrophys. Cosmol. 3, 149 (1987).

[3] A. H. Guth, Phys. Rev. D 23, 347 (1981); Adv. Ser. Astrophys. Cosmol. 3, 139 (1987).

[4] A. A. Starobinsky, JETP Lett. 30, 682 (1979); 30, 767 (1979).

[5] U. Seljak and M. Zaldarriaga, Phys. Rev. Lett. 78, 2054 (1997).

[6] D. H. Lyth, Phys. Rev. Lett. 78, 1861 (1997).

[7] D. N. Spergel et al. (WMAP Collaboration), Astrophys. J. Suppl. 170, 377 (2007).

[8] P. A. R. Ade et al. (Planck Collaboration), Astron. Astrophys. 594, A20 (2016).

[9] D. J. Schwarz, Mod. Phys. Lett. A 13, 2771 (1998).
[10] M. Maggiore, Phys. Rep. 331, 283 (2000).

[11] A. Mazumdar and G. White, arXiv:1811.01948.

[12] N. Seto, S. Kawamura, and T. Nakamura, Phys. Rev. Lett. 87, 221103 (2001).

[13] S. Sato et al., J. Phys. Conf. Ser. 840, 012010 (2017).

[14] H. Audley et al. (LISA Collaboration), arXiv:1702.00786.

[15] G. Janssen et al., Proc. Sci., AASKA14 (2015) 037.

[16] L. Lentati et al., Mon. Not. R. Astron. Soc. 453, 2576 (2015).

[17] Y. Watanabe and E. Komatsu, Phys. Rev. D 73, 123515 (2006).

[18] S. Schettler, T. Boeckel, and J. Schaffner-Bielich, Phys. Rev. D 83, 064030 (2011).

[19] C. Caprini and D. G. Figueroa, Classical Quantum Gravity 35, 163001 (2018).

[20] Y. Aoki, G. Endrodi, Z. Fodor, S. D. Katz, and K. K. Szabo, Nature (London) 443, 675 (2006). 
[21] M. Drees, F. Hajkarim, and E. R. Schmitz, J. Cosmol. Astropart. Phys. 06 (2015) 025.

[22] P. Castorina, D. Lanteri, and S. Mancani, Phys. Rev. D 98, 023007 (2018).

[23] S. Anand, U. K. Dey, and S. Mohanty, J. Cosmol. Astropart. Phys. 03 (2017) 018.

[24] D. J. Schwarz, Ann. Phys. (Amsterdam) 12, 220 (2003).

[25] M. Stuke, D. J. Schwarz, and G. Starkman, J. Cosmol. Astropart. Phys. 03 (2012) 040.

[26] K. Saikawa and S. Shirai, J. Cosmol. Astropart. Phys. 05 (2018) 035.

[27] M. Hindmarsh and O. Philipsen, Phys. Rev. D 71, 087302 (2005).

[28] S. Borsanyi et al., Nature (London) 539, 69 (2016).

[29] S. Capozziello, M. Khodadi, and G. Lambiase, Phys. Lett. B 789, 626 (2019).

[30] M.-W. Li, Y. Yang, and P.-H. Yuan, arXiv:1812.09676.

[31] E. Witten, Phys. Rev. D 30, 272 (1984).

[32] M. Asakawa and K. Yazaki, Nucl. Phys. A504, 668 (1989).

[33] D. J. Schwarz and M. Stuke, J. Cosmol. Astropart. Phys. 11 (2009) 025; 10 (2010) E01(E).

[34] S. Dodelson, Modern Cosmology (Academic Press, Amsterdam, 2003).

[35] M. Cheng et al., Phys. Rev. D 77, 014511 (2008).

[36] A. Bazavov et al. (HotQCD Collaboration), Phys. Rev. D 90, 094503 (2014).

[37] A. Bazavov et al., Phys. Rev. D 95, 054504 (2017).

[38] If one does not solve Eq. (6) in this way, it leads to an error in the computed PGW spectrum which can be larger than the effects from the QCD transition. Basically, since the energy density of radiation has changed for a specific scale factor with respect to today, it leads to a shift in the amplitude for a given frequency.

[39] S. Weinberg, Phys. Rev. D 69, 023503 (2004).

[40] The energy and entropy density can also be written as a function of degrees of freedom, i.e., $\rho_{\text {tot }}(T, \mu)=$ $\left(\pi^{2} / 30\right) g_{\rho, \text { tot }}(T, \mu(T)) T^{4} \quad$ and $\quad s_{\text {tot }}(T, \mu)=\left(2 \pi^{2} / 45\right) g_{s, \text { tot }}$ $(T, \mu(T)) T^{3}$.

[41] M. Laine and Y. Schroder, Phys. Rev. D 73, 085009 (2006).
[42] M. Laine and M. Meyer, J. Cosmol. Astropart. Phys. 07 (2015) 035.

[43] J. Lesgourgues and S. Pastor, Adv. High Energy Phys. 2012, 608515 (2012).

[44] J. O. Andersen, L. E. Leganger, M. Strickland, and N. Su, Phys. Lett. B 696, 468 (2011).

[45] K. Kajantie, M. Laine, K. Rummukainen, and Y. Schroder, Phys. Rev. D 67, 105008 (2003).

[46] P. Huovinen and P. Petreczky, Nucl. Phys. A837, 26 (2010).

[47] S. Borsanyi, G. Endrodi, Z. Fodor, A. Jakovac, S. D. Katz, S. Krieg, C. Ratti, and K. K. Szabo, J. High Energy Phys. 11 (2010) 077.

[48] A. Gynther and M. Vepsalainen, J. High Energy Phys. 01 (2006) 060.

[49] M. D’Onofrio and K. Rummukainen, Phys. Rev. D 93, 025003 (2016).

[50] D. Buttazzo, G. Degrassi, P. P. Giardino, G. F. Giudice, F. Sala, A. Salvio, and A. Strumia, J. High Energy Phys. 12 (2013) 089.

[51] P. F. de Salas and S. Pastor, J. Cosmol. Astropart. Phys. 07 (2016) 051.

[52] I. M. Oldengott and D. J. Schwarz, Europhys. Lett. 119, 29001 (2017).

[53] N. Aghanim et al. (Planck Collaboration), arXiv:1807.06209.

[54] J. A. Harvey and M. S. Turner, Phys. Rev. D 42, 3344 (1990).

[55] M. M. Wygas, I. M. Oldengott, D. Bödeker, and D. J. Schwarz, Phys. Rev. Lett. 121, 201302 (2018).

[56] A. Bazavov et al., arXiv:1812.08235.

[57] M. Wygas, Ph. D. thesis, University of Bielefeld, 2018.

[58] C. Gebhardt, Bachelor thesis, Goethe University, Frankfurt, 2018.

[59] A. Bazavov et al., Phys. Lett. B 737, 210 (2014).

[60] S. Mukherjee, P. Petreczky, and S. Sharma, Phys. Rev. D 93, 014502 (2016).

[61] H. Abuki, T. Brauner, and H. J. Warringa, Eur. Phys. J. C 64, 123 (2009).

[62] B. B. Brandt, G. Endrodi, E. S. Fraga, M. Hippert, J. Schaffner-Bielich, and S. Schmalzbauer, Phys. Rev. D 98, 094510 (2018). 